\title{
Effects of immunisation against PCSK9 in mice bearing melanoma
}

\author{
Amir Abbas Momtazi-Borojeni ${ }^{1,2}$, Maryam Ebrahimi Nik³, Mahmoud Reza Jaafari ${ }^{4,5}$, \\ Maciej Banach ${ }^{6,7}$, Amirhossein Sahebkar ${ }^{5,8,9}$
}

\author{
${ }^{1}$ Nanotechnology Research Center, Bu-Ali Research Institute, Mashhad University \\ of Medical Sciences, Mashhad, Iran \\ ${ }^{2}$ Department of Medical Biotechnology, Student Research Committee, \\ Faculty of Medicine, Mashhad University of Medical Sciences, Mashhad, Iran \\ ${ }^{3}$ Nanotechnology Research Center, Student Research Committee, Pharmaceutical \\ Technology Institute, Mashhad University of Medical Sciences, Mashhad, Iran \\ ${ }^{4}$ Nanotechnology Research Center, Pharmaceutical Technology Institute, \\ Mashhad University of Medical Sciences, Mashhad, Iran \\ ${ }^{5}$ Biotechnology Research Center, Pharmaceutical Technology Institute, \\ Mashhad University of Medical Sciences, Mashhad, Iran \\ ${ }^{6}$ Department of Hypertension, WAM University Hospital in Lodz, Medical University \\ of Lodz, Lodz, Poland \\ 'Polish Mother's Memorial Hospital Research Institute (PMMHRI), Lodz, Poland \\ ${ }^{8}$ Neurogenic Inflammation Research Center, Mashhad University of Medical Sciences, \\ Mashhad, Iran \\ ${ }^{9}$ School of Pharmacy, Mashhad University of Medical Sciences, Mashhad, Iran
}

Submitted: 20 April 2019

Accepted: 16 July 2019

Arch Med Sci 2020; 16 (1): 189-199

DOI: https://doi.org/10.5114/aoms.2020.91291

Copyright $\odot 2019$ Termedia \& Banach

\begin{abstract}
Introduction: Inhibition of proprotein convertase subtilisin/kexin 9 (PCSK9) is an established modality for the treatment of hypercholesterolaemia. However, the impact of PCSK9 inhibition in other situations such as cancer remains largely unknown. The current study was conducted to study the effects of PCSK9 inhibition on cancer endpoints in mice bearing melanoma. Material and methods: To generate antiPCSK9 antibody in vivo, a nanoliposomal antiPCSK9 vaccine adsorbed to $0.4 \%$ Alum adjuvant was subcutaneously injected in C57BL/6 mice four times with bi-weekly intervals. Two weeks after the last immunisation, mice were subcutaneously inoculated with B16F0 melanoma cells. After a tumour mass was palpable (approximately $10 \mathrm{~mm}^{3}$ ), the mice were randomly divided into four groups and subjected to different treatment protocols: (1) PBS (untreated control), (2) vaccine group, (3) the combination of vaccine and a single dose of liposomal doxorubicin $\left(\right.$ Doxil $\left.^{\oplus}\right)$, and (4) liposomal doxorubicin (positive control) group. To determine therapeutic efficacy, mouse body weight, tumour size, and survival were monitored every three days for 36 days.

Results: The nanoliposomal antiPCSK9 vaccine was found to efficiently induce specific antibodies against PCSK9 in C57BL/6 mice, thereby reducing plasma levels and function of PCSK9. Tumour volumes in the vaccinated group were not significantly different from those in the liposomal doxorubicin, combination, and control groups. The time to reach endpoint (TTE) values of the vaccine ( $28 \pm 5$ days), combination ( $30 \pm 6$ days), liposomal doxorubicin ( $34 \pm 2$ days), and control ( $31 \pm 2$ days) groups were not significantly different, either. Furthermore, the tumour growth delay (TGD) values of the vaccine $(-11.5 \pm 15.4 \%)$, liposomal doxorubicin $(7.75 \pm 6.5 \%)$, combination $(-6 \pm 20.77 \%)$, and control $(0 \pm 7.5)$ groups were not significantly different. Finally, there was no significant difference between the median survival time and lifespan of the vaccinated versus other tested groups.
\end{abstract}

\author{
Corresponding author: \\ Amirhossein Sahebkar \\ Biotechnology Research \\ Center \\ Pharmaceutical Technology \\ Institute \\ Mashhad University \\ of Medical Sciences \\ Mashhad 9177948564, Iran \\ E-mail: sahebkara@mums. \\ ac.ir, \\ amir_saheb2000@yahoo.com
}


Conclusions: The nanoliposomal PCSK9 vaccine did not adversely affect the growth of melanoma tumour nor the survival of tumour-bearing mice.

Key words: cancer, liposome, immunisation, melanoma, nanoparticle, PCSK9, vaccine.

\section{Introduction}

Investigations into melanoma and other cancer types have suggested a potential role for dysregulated cholesterol homeostasis in the development of cancer [1-8]. Experimental studies have shown that a high-fat diet enhances tumour growth and metastasis of xenograft human melanoma cells $[9,10]$. Cholesterol can activate cancer-related signalling pathway, e.g. the hedgehog pathway, through direct binding to the G-protein-coupled receptors, including smoothened receptor $[11,12]$ and adenosine A2A receptor [13], by which affect cell differentiation and proliferation as well as tumour formation [14]. Cholesterol can also activate other oncogenic signalling pathways through binding to the scaffold proteins such as NHERF1/ EBP50 [15], which regulate oncogenic signalling networks via assembling cancer-associated proteins, including those involved in the $\mathrm{Wnt} / \beta$-catenin and PI3K/Akt pathways [16].

Although disruption of cholesterol homeostasis has been proposed to contribute to cancer development, the potential of therapeutic targeting of cholesterol homeostasis is a debatable issue in oncology. Previous studies of lipid-lowering drugs have shown an association between cancer risk and low levels of low-density lipoprotein (LDL) cholesterol (LDL-C) [17]; however, extant evidence suggests that the drugs per se do not enhance cancer risk [18-21].

Proprotein convertase subtilisin/kexin 9 (PCSK9) is an important modulator of cholesterol haemostasis through controlling the clearance of the plasma LDL-C from the bloodstream via regulation of the liver's LDL receptor (LDLR). PCSK9 was found to be a therapeutic target for lowering LDL-C since early studies showed that gain-offunction mutations in PCSK 9 are causally associated with the elevated plasma level of LDL-C [22], while loss-of-function mutations are associated with hypocholesterolaemia and a decreased risk of coronary artery disease [23-26]. Although scientific evidence supports the benefits of PCSK9 inhibitors in lowering LDL-C and reducing cardiovascular outcomes [27-33], the lipid-independent effects of PCSK9 inhibition, especially in patients with cancer, has remained largely unexplored. Hitherto, there have been several human studies that evaluated a possible link between PCSK 9 polymorphisms and the risk of cancer, and the results have been contradictory [34-36]. A previ- ous study could statistically associate LDL-raising PCSK9 variants with a higher risk of the cancer [34], while another study could not validate an association between PCSK9 loss-of-function variants and increased cancer incidence [35]. In contrast to these reports, a recent Mendelian-randomisation study showed that PCSK9 LDL-raising variants are associated with a higher risk of cancer, while LDL-lowering polymorphisms mimicking PCSK9 inhibitors were reported to be associated with a reduced risk of cancer occurrence [36]. These inconsistent results necessitate additional studies to evaluate the efficacy and safety of PCSK9 inhibitors in cancer.

Anti-PCSK9 vaccines are emerging PCSK9 inhibitors in the pipeline, which have shown significant LDL-lowering effects in experimental models of hypercholesterolaemia [37-40]. We previously formulated a nanoliposomal anti-PCSK9 vaccine that could efficiently promote long-lasting, specific, and safe anti-PCSK9 antibodies in BALB/C mice. Nanoliposomal vaccine-induced antibodies were found to target plasma PCSK9 and suppress its binding to LDLR, thereby leading to the inhibition of PCSK9 function [41]. The present study was carried out to assess the effects of the mentioned nanoliposomal anti-PCSK9 vaccine in C57 $\mathrm{BL} / 6$ mice bearing B16F0 melanoma.

\section{Material and methods}

\section{Vaccine preparation and characterisation}

\section{Preparation and characterisation of the liposome nanoparticles}

The thin-film lipid hydration method was employed to manufacture nanoliposome formulation containing 1,2-Dimyristoyl-sn-glycero-3-phosphorylcholine (DMPC), 1,2-dimyristoyl-sn-glycero-3phosphorylglycerol (DMPG), and cholesterol (Chol) (Avanti Polar Lipid; Alabaster, USA) at the final concentration of $40 \mathrm{mM}$ (total phospholipids and Chol). In brief, DMPC, DMPG, and Chol were mixed in chloroform at the molar ratios of $75: 10: 15$, respectively. Lipid mixture was dried to a thin lipid film under decreased pressure by rotary evaporation (Heidolph, Germany). Then, the obtained lipid-film was freeze-dried (VD-800F, Taitech, Japan) overnight to entirely eliminate the organic solvent. Afterward, the dried lipids were hydrated with $10 \mathrm{mM}$ HEPES buffer ( $\mathrm{pH}$ 7.2) containing $5 \%$ dextrose, and vortexed and bath-sonicated 
to disperse completely into the buffer. To obtain small unilamellar vesicles (SUVs) with a uniform size of $100 \mathrm{~nm}$, the multilamellar vesicles (MLVs) were extruded using a mini extruder (Avestin, Canada) with polycarbonate membranes of 600 , 400, 200, and $100 \mathrm{~nm}$ pore size, respectively. Particle size (diameter, nm), zeta potential (surface charge, $\mathrm{mV}$ ), and poly dispersity index (PDI) of the prepared nanoliposomal formulation were determined using dynamic light scattering (DLS) technique on a Zetasizer (Nano-ZS,Malvern, UK) at room temperature (RT). The synthesised nanoliposomes were stored at $4^{\circ} \mathrm{C}$ under argon.

\section{Construction of immunogenic peptide}

The immunogenic fused PCSK9-tetanus (IFPT) peptide with a purity grade of $>95 \%$ was synthesised and high-performance liquid chromatography (HPLC)-purified by ChinaPeptides Co., Ltd. (Shanghai, China). Already designed, the IFPT peptide [42] includes a PCSK9 sequence as a $B$ cell epitope inspired from the AFFiRiS group $[43,44]$, and a T-helper cell epitope belonging to tetanus toxin used as a pharmaceutically acceptable adjuvant carrier [45] (Table I). To conjugate IFPT epitope on the surface of liposome nanoparticles, it was linked to DSPE-PEG-Mal (1,2-distearoyl-sn-glycero-3-phosphoethanolamine- $N$-[maleimide(PEG)-2000]) lipid (Lipoid GmbH, Germany) via an N-terminal cysteine residue added to IFPT peptide (Figure 1).

\section{Construction of DSPE-PEG-IFPT micelles}

DSPE-PEG-Maleimide lipid was employed to attach the immunogenic peptides on the surface of liposome nanoparticles as an adjuvant delivery system. N-terminal cysteine residue of the IFPT peptide provides a thiol group that reacts with pyrrole group maleimide and produces a thioether bond; thereby, the peptide covalently links to DSPE-PEG-Maleimide lipid. The IFPT peptide and DSPE-PEG-Mal at the molar ratios of $1.2: 1$, respectively, were mixed in DMSO/chloroform solution at the volume ratio of $1: 1$ and then slowly stirred at RT for $48 \mathrm{~h}$. The linkage was verified by TLC (thin layer chromatography) method with the mobile phase containing chloroform, methanol, and water at the volume ratio of $90: 18: 2$. Then, the DMSO/chloroform solution was dried by rotary evaporator and freeze-drying followed by hydration with sterile deionised water $(\mathrm{pH} \mathrm{7.2)}$ at $30^{\circ} \mathrm{C}$ to construct DSPE-PEG-IFPT micelles. The efficiency of the linkage in the prepared micelles was determined by HPLC analysis. The true value of the linked micelles was determined using efficiency of linkage and content of total lipid measured by the Bartlett phosphate assay method [46].

\section{HPLC analysis of the linkage efficiency}

The efficiency of the linkage between the IFPT peptide and DSPE-PEG-Mal linker was indirectly measured by HPLC quantification of the free

Table I. Sequence of the immunogenic peptides used in the present study

\begin{tabular}{|lcc|}
\hline Peptide name & Sequence & Immunogenicity \\
\hline PCSK9 & S-I-P-W-N-L-E-R-I-T-P-V-R & B cell epitope \\
\hline Tetanus & A-Q-Y-I-K-A-N-S-K-F-I-G-I-T-E-L & T cell epitope \\
\hline IFPT & ${ }^{*}$ CGGGSIPWNLERITPVRAQYIKANSKFIGITEL & \\
\hline
\end{tabular}

${ }^{\star}$ The bold amino acid codes are as a linker sequence for conjugating with DSPE-PEG-Mal. IFPT-immunogenic fused PCSK9-tetanus.

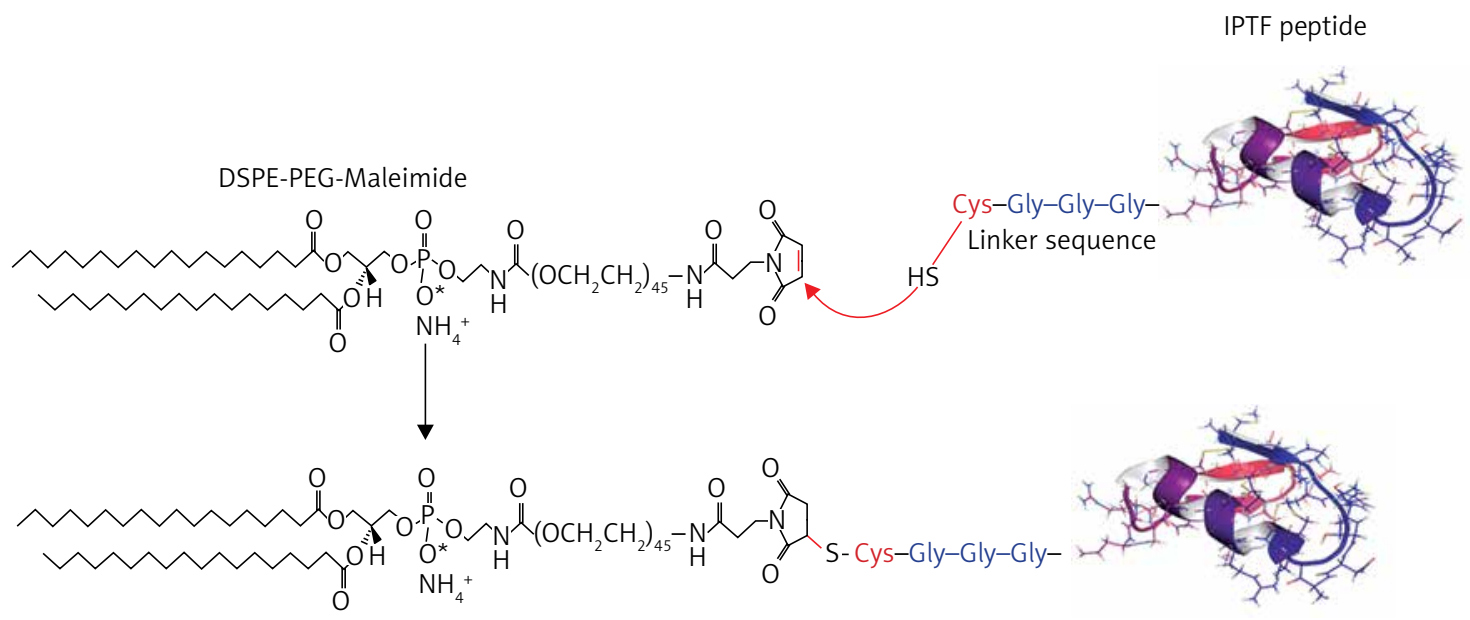

Figure 1. Schematic view of linking between peptide and DSPE-PEG-Maleimide 
peptide content of the prepared DSPE-PEG-IFPT micelles. The HPLC apparatus was equipped with a Smart Line HPLC Pump 1000, a PDA Detector 2800 (set at $220 \mathrm{~nm}$ ), and a Degasser5000, all from Knauer (Berlin, Germany). Each sample $(20 \mu \mathrm{l})$ was injected through a Smart Line Auto Sampler, and data were obtained and processed with ChromGate software (version 3.3.1) from Knauer (Berlin, Germany). Chromatographic separation was performed on a C18 reverse-phase column, $4.6 \mathrm{~mm} \times 25 \mathrm{~cm}$ (Shimadzu, Japan), using an isocratic mobile phase of ( $0.1 \%$ TFA in water $) /(0.1 \%$ TFA in acetonitrile) at gradient ratios of $55 / 45$ to $45 / 55$ in $10 \mathrm{~min}$, at a flow rate of $1 \mathrm{ml} / \mathrm{min}$.

The IFPT peptide with HPLC purity $>95 \%$ was used as a standard solution. The free peptide peak within the chromatogram of the micelle sample was identified and assigned based on the retention time $(2.2 \mathrm{~min})$ of the standard solution, followed by sample spiking. To quantify the free peptide content of the micelle sample, a calibration curve was constructed by injecting standard solution at five concentrations $(50-500 \mu \mathrm{g} / \mathrm{ml})$, which was linear, with a correlation coefficient $\left(r^{2}\right)$ of 0.9954 . Using linear regression analysis of the calibration curve appearing in the standard chromatogram, the free peptide content of the micelle sample was estimated. Linkage efficiency in the constructed DSPE-PEG-IFPT micelles was calculated by subtracting the free peptide amount within the micelles quantified by HPLC from the amount of the IFPT peptide initially added.

\section{Construction and characterisation of nanoliposomal IFPT vaccine}

Nanoliposomes were used as a delivery adjuvant to enhance immunogenicity of the peptide. Because many IFPT peptides can be conjugated to the surface of liposome nanoparticles, we propose that IFPT-linked nanoliposomes can elicit a high-titre antibody against self-antigen PCSK9, maybe through elevating peptide valency. To conjugate the IFPT peptide on the nanoliposome surface, the post insertion approach was performed, in which the prepared DSPE-PEG-IFPT micelles $(100 \mu \mathrm{g}$, based on the linked peptide) and liposome nanoparticles
$(1 \mathrm{ml})$ were mixed and then gently shaken at $45^{\circ} \mathrm{C}$ for $3 \mathrm{~h}$. The micelles were inserted in the nanoliposome bilayer via DSPE phospholipid moiety, and expose IFPT peptides on the nanoliposome surface through the PEG chains. Particle size, surface charge, and PDI of the prepared nanoliposomal IFPT particles were evaluated using DLS technique on a Zetasizer (Nano-ZS, Malvern, UK) at RT. The IFPT-conjugated nanoliposomes were adsorbed to $0.4 \%$ Alum adjuvant (Sigma-Aldrich) at a $1: 1$ (v:v) ratio in a total volume of $400 \mu \mathrm{l}$ and stored at $4^{\circ} \mathrm{C}$ under argon. Prior to injection, the nanoliposomal IFPT plus Alum vaccine, hereafter called L-IFPTA ${ }^{+}$, was brought to RT and carefully mixed.

\section{Animal and cell line}

A total of 20 female C57BL/6 mice (4-6 weeks old) were bought from the Pasteur Institute of Tehran, Iran and fed with ad libitum access to purified water and a commercial stock diet. All mice were housed in a pathogen-free animal house at a temperature of $22 \pm 1^{\circ} \mathrm{C}$ with a 12 : 12-h light: dark cycle and maintained under a relative humidity of $50 \pm 10 \%$. Animal care was performed in accordance with welfare guidelines established by the Institutional Ethical Committee and Research Advisory Committee of Mashhad University of Medical Sciences. At the end of the experiment all animals were euthanised by $\mathrm{CO}_{2}$ inhalation.

The B16F0 melanoma cell line was provided from the Pasteur Institute of Tehran, Iran and cultured in RPMI-1640 medium containing 10\% FBS and supplemented with penicillin $(100 \mathrm{lU} / \mathrm{ml}) /$ streptomycin $(100 \mathrm{mg} / \mathrm{ml})$. The cells were incubated at $37^{\circ} \mathrm{C}$ with a $5 \% \mathrm{CO}_{2} / 95 \%$ air humidified atmosphere.

\section{Vaccination program}

Following one week of taming prior to the experimental procedures, the mice were randomly arranged into two groups: a vaccine group $(n=10)$ and an untreated group $(n=10)$. The vaccination was primed at week 0 (W0) and followed by three boosters (W2, W4, and W6), in a bi-weekly interval via subcutaneous administration (Figure 2), while untreated mice simultaneously received phosphate-buffered saline (PBS). The tail vein bleeding

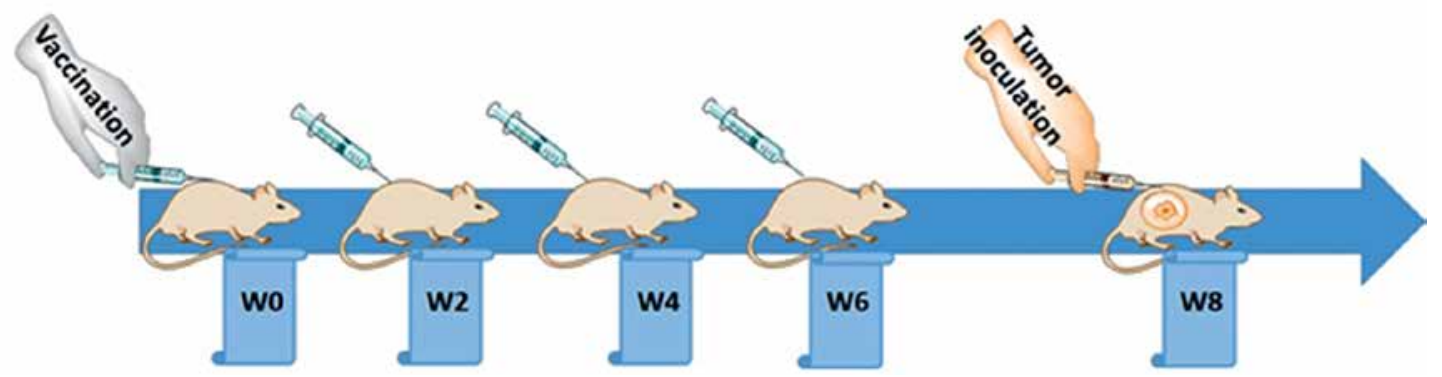

Figure 2. A summary of study design 
was performed 2 weeks after each vaccination for the titration of plasma anti-PCSK9 antibody.

\section{ELISA measuring of plasma anti-PCSK9 peptide antibody}

To determine the titre of anti-PCSK9 antibodies, plasma samples were collected and analysed by ELISA method. Briefly, PCSK9 peptide at the concentration of $5 \mu \mathrm{g} / \mathrm{ml}$ in $0.1 \mathrm{M} \mathrm{NaHCO}_{3}$ (pH 9.2-9.4) was coated overnight in a 96-well Nunc-MaxiSorp plate. Free binding sites were then blocked by the incubation with blocking buffer $(1 \times \mathrm{PBS}, 1 \% \mathrm{BSA})$ for $1 \mathrm{~h}$ at $37^{\circ} \mathrm{C}$. Diluted plasma (1 : 400 in $1 \times$ PBS/0.1\% BSA/0.05\% Tween-20) was added, serially diluted $1: 4$, and incubated for $1 \mathrm{~h}$ at $37^{\circ} \mathrm{C}$. Each ELISA plate contained a standard antibody as an internal control. For the detection, biotinylated anti-mouse IgG $(\mathrm{H}+\mathrm{L})$ (Sigma-Aldrich; 1 : 1000) in $1 \times \mathrm{PBS} / 0.1 \% \mathrm{BSA} / 0.1 \%$ Tween-20 was applied and incubated for $1 \mathrm{~h}$ at $37^{\circ} \mathrm{C}$. As a next step, horseradish peroxidase coupled to streptavidin (Roche) was added (30 min, $37^{\circ} \mathrm{C}$ ) followed by the addition of the substrate 2,2'-Azinobis [3-ethylbenzothiazoline-6-sulfonic acid]-diammonium salt (ABTS) (Sigma-Aldrich) (15 min, RT). The optical density (OD) at $450 \mathrm{~nm}$ was measured with a microwell plate reader (Sunrise, Tecan, Switzerland), and the titres were defined as the dilution factor referring to $50 \%$ of the maximal optical density $\left(\mathrm{OD}_{\max } / 2\right)$. The mean titres \pm SD of all animals per group are presented.

\section{Plasma PCSK9 quantification}

Plasma PCSK9 concentration in the vaccinated mice was measured by CircuLex rat PCSK9 ELISA (CircuLexTM, Cy-8078, MBL, Woburn, MA) according to the manufacturer's instructions. Briefly, $100 \mu \mathrm{l}$ of the diluted $1: 100$ plasma samples was added on a 96-well microplate and incubated for $1 \mathrm{~h}$ at RT. A HRP-conjugated anti-PCSK9 antibody was added for $1 \mathrm{~h}$ followed by the substrate reagent and stop solution, all at RT. Optical density was detected at $450 \mathrm{~nm}$ with a Microwell plate reader (Sunrise, Tecan, Switzerland). A standard curve provided by the supplier was defined to measure PCSK9 concentration.

\section{PCSK9 inhibition analysis}

To evaluate inhibition of mice PCSK9 by vaccine-induced antibodies, interaction of generated-antibodies with PCSK9 was assayed. For this purpose, the same kit Circulex rat PCSK9 ELISA was used, but in turn of HRP-conjugated anti-PCSK9 antibody, detection was performed with HRP-conjugated anti-mouse IgG $(\mathrm{H}+\mathrm{L})$ (Sigma Aldrich; dilution 1 : 5000) incubated for $1 \mathrm{~h}$ at RT, followed by the substrate reagent and stop solu- tion provided by the supplier. The OD was detected at $450 \mathrm{~nm}$ with the Microwell plate reader.

\section{In vitro evaluation of inhibiting PCSK9-LDLR interaction}

CircuLex PCSK9-LDLR in vitro binding assay kit (CircuLex ${ }^{\top M}$, Cy-8150, MBL, Woburn, MA) was employed to analyse the ability of vaccine-generated antibodies for inhibition of the PCSK9-LDLR interaction in vitro. Briefly, $100 \mu$ l of vehicle control or the plasma samples of vaccinated mice was added to a 96-well microplate pre-coated with a recombinant LDLR-AB domain, which contained binding site for PCSK9. Immediately after that, the reaction was initiated by adding a "His-tagged PCSK9 wild type" solution incubated for $2 \mathrm{~h}$ followed by adding a biotinylated anti-His-tag monoclonal antibody for $1 \mathrm{~h}$ at RT. Then, HRP-conjugated streptavidin was coated for 1-h at RT followed by the substrate reagent and stop solution. In this method, the higher amount of PCSK9-LDLR interaction is associated with higher ELISA OD, in which, in the presence of anti-PCSK9 antibody, this interaction is inhibited and consequently ELISA OD is decreased. A dose-response curve with appropriate serial dilutions of "His-tagged PCSK9 wild type" solution was drawn to measure accurate inhibition percentage of test samples.

\section{Evaluation of in vivo anti-tumour efficacy}

Two weeks after the last booster, the vaccinated and unvaccinated C57BL/6 mice were subcutaneously inoculated with B16F0 melanoma cells $\left(5 \times 10^{5} / 50 \mu \mathrm{l} \mathrm{PBS} /\right.$ mouse $)$ into the right flank at day zero. Tumour growth was monitored in a threeday interval by calculating the tumour volume after measuring three orthogonal diameters with callipers according to the formula: Tumour volume $\left(\mathrm{mm}^{3}\right)=($ length $\times$ height $\times$ width $) \times 0.52$. After the tumour mass was palpable (approximately $10 \mathrm{~mm}^{3}$ ) on day 10 , the mice were randomly divided into four groups (5 mice/group) and subjected to different treatment protocols: (1) PBS (untreated control) group, which comprised unvaccinated tumour-bearing mice receiving single tail vein injection of saline buffer; (2) vaccine group, which comprised vaccinated tumour-bearing mice receiving a single tail vein injection of saline buffer; (3) the combination group which comprised vaccinated tumour-bearing mice receiving a single tail vein injection of Doxil (15 mg/kg); and (4) the Doxil (positive control) group, which comprised unvaccinated tumour-bearing mice receiving a single tail vein injection of Doxil (15 mg/kg).

To evaluate therapeutic efficacy, mouse body weight, tumour size, and survival were monitored every 3 days for 38 days. Animals euthanasia 
Table II. Physical properties of nanoliposomal formulations

\begin{tabular}{|lccc|}
\hline Formulation & $\begin{array}{c}\text { Z-average }[\mathrm{nm}] \\
\text { Mean } \pm \mathrm{SD}, \boldsymbol{n = 3}\end{array}$ & $\begin{array}{c}\text { Zeta potential [mV] } \\
\text { Mean } \pm \text { SD, } n=3\end{array}$ & $\begin{array}{c}\text { PDI } \\
\text { Mean } \pm \text { SD, } n=3\end{array}$ \\
\hline The empty nanoliposome & $140 \pm 8$ & $-45 \pm 3$ & $0.08 \pm 0.01$ \\
\hline The IFPT-linked nanoliposome & $170 \pm 6$ & $-30 \pm 2$ & $0.1 \pm 0.08$ \\
\hline
\end{tabular}

$P D I$ - polydispersity index.

$\left(\mathrm{CO}_{2}\right.$ inhalation) occurred on those with 4T1 tumour due to the following ethical considerations: body weight loss $>20 \%$ of initial mass, tumour volume greater than $2.0 \mathrm{~cm}$ in one dimension, or if the mice became sick and unable ambulate to reach food/water $[47,48]$. For each mouse, the time to reach tumour volume above $1000 \mathrm{~mm}^{3}$ or the time to reach end point (TTE) as a response variable was calculated from the equation of the line obtained by exponential regression of the tumour growth curve. For each group, the percent of tumour growth delay (\%TGD) was determined by calculating the difference between the average TTE of treatment group ( $\mathrm{T})$ and the average TTE of the control group $(C),(\% T G D=[(T-C) / C] \times 100)$ [49]. For each treatment group, the percent of increased life span (\%ILS) was measured based on the following formula: [(the average survival time of treatment group/the average survival time of control group $\times 100)-100]$ [50].

\section{Statistical analysis}

Statistical analysis was performed using SPSS Statistics version 20 and GraphPad Prism version

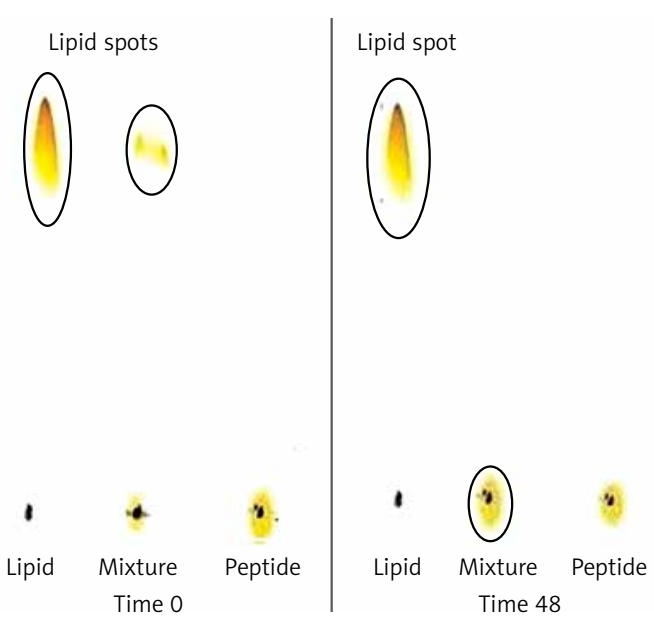

Figure 3. Assessment of conjugation between DSPE-PEG-Mal and the IFPT peptide at the time zero and $48 \mathrm{~h}$ after starting of reaction. Lipid (DSPEPEG-Mal) is dissolved in the mobile phase and ascends to the top of the TLC plate (spots in the top of the left and middle lines), but peptide is bound to the silica and remains in the spotting point (the middle line). After 48, lipid bound to peptide and stayed in the point of spotting, and therefore the lipid spot on the top of the reaction mixture line disappeared, indicating the conjugation of the IFPT peptide and DSPE-PEG-Mal linker
7.04 software. Survival data were analysed using the log-rank (Mantel-Cox) test. Other comparisons were done using one-way ANOVA and Tukey post-hoc multiple comparison test. Values were expressed as mean \pm SD or median for normally and non-normally distributed data, respectively. Results with $p<0.05$ were considered as statistically significant.

\section{Results}

\section{Characterisation of nanoliposomes}

Physical properties of the free and IFPT-linked nanoliposomes are shown in Table II. The free and IFPT-linked nanoliposomes had a size range from $140 \mathrm{~nm}$ to $170 \mathrm{~nm}$ in diameter, with polydispersity index values of $<0.2$, showing homogeneity of particles. Zeta potential measurement indicated negative charge on the surface of nanoparticles.

\section{TLC and HPLC analysis of DSPE-PEG-IFPT micelles}

Conjugation of DSPE-PEG-Mal linker and the IFPT peptide was confirmed by evaluating the extinction of DSPE-PEG-Mal spot (lipid spot) on the TLC plate, following a 48-h reaction (Figure 3). Afterward, DSPE-PEG-IFPT micelles were prepared, and the efficiency of conjugation was measured using HPLC analysis, indirectly, by quantification of the free peptide content of the micelle sample. HPLC results indicated the conjugation of $96 \%$ of the initial IFPT peptides to DSPE-PEG-Mal linker (Figure 4).

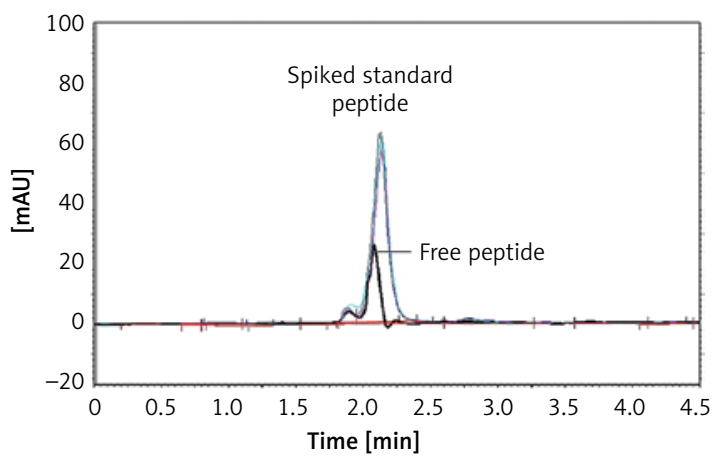

Figure 4. HPLC chromatogram of DSPE-PEG-IFPT micelles and reference standard IFPT peptide. The retention time of reference standard was observed at $2.2 \mathrm{~min}$, and it was found to be the same with free peptide present in the micelle sample 
Induction of PCSK9 antibodies in C57BL/6 mice by L-IFPTA ${ }^{+}$vaccine

IFPT-exposing nanoliposomal vaccine was found to induce high titres IgG antibody against PCSK9 peptide in C57BL/6 mice upon four vaccinations in biweekly intervals. Further, long-term analysis revealed that anti-PCSK9 antibody titre peaked at week 8 and remained constant up to week 10, and then exhibited a diminishing trend (Figure $5 \mathrm{~A}$ ).

\section{Specific targeting of plasma PCSK9 vaccine- induced PCSK9 antibodies}

L-IFPT vaccine provoked antibodies that could directly and specifically target plasma PCSK9 in C57BL/6 mice. Plasma levels of PCSK9 in the vaccine and control groups were $17 \pm 5 \mathrm{ng} / \mathrm{ml}$ and $58 \pm 9 \mathrm{ng} / \mathrm{ml}$, respectively (Figure $5 \mathrm{~B}$ ). Compared with the control mice, plasma levels of PCSK 9 was significantly decreased by $70 \%(-41 \pm 6 \mathrm{ng} / \mathrm{ml}, p=$ 0.009 ) in the vaccinated mice. To evaluate specific targeting, PCSK9 inhibition was evaluated using
ELISA-based assay to detect vaccine-induced antibodies directly bound to plasma PCSK9. Therefore, plasma PCSK9 obtained from the vaccinated and control mice was captured onto murine antiPCSK9 antibodies-coated ELISA plates, and vaccine-induced PCSK9-bound murine antibodies were detected using an anti-mouse IgG antibody. As shown in Figure $5 \mathrm{C}$, the significantly higher $\mathrm{OD}_{450}$ signal indicated from plasma of vaccinated mice revealed a direct binding of vaccine-generated anti-PCSK9 antibodies to PCSK9.

\section{PCSK9-LDLR binding inhibition \\ by vaccine-induced PCSK9 antibodies}

In vitro PCSK9-LDLR binding assay revealed that in the presence of plasma anti-PCSK9 antibodies, interaction between murine PCSK9 and LDLR was inhibited. Plasma obtained from the L-IFPTA ${ }^{+}$group could significantly decrease PCSK9 binding to LDLR by $50 \%$, compared with the plasma from the control group (Figure $5 \mathrm{D}$ ).
A

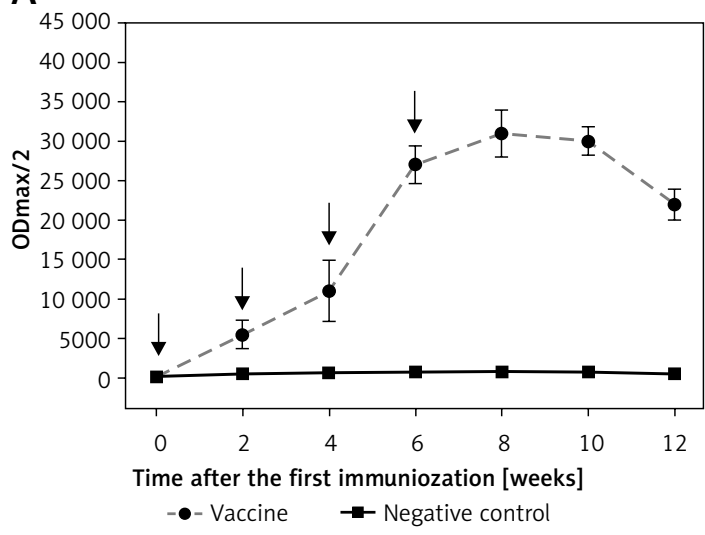

C

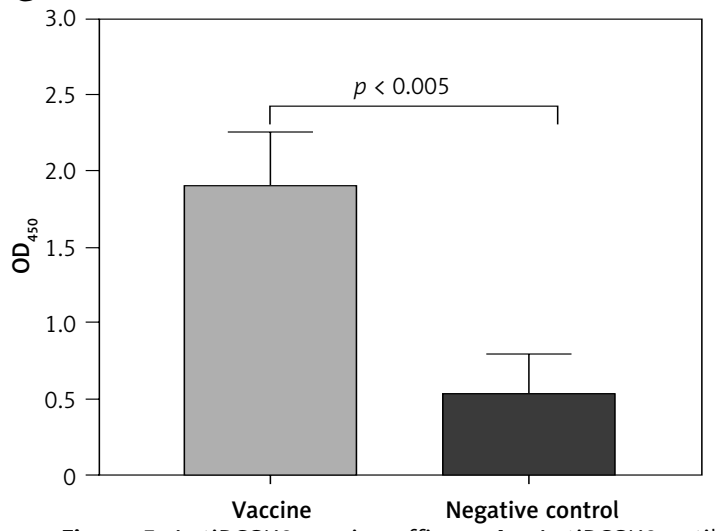

B

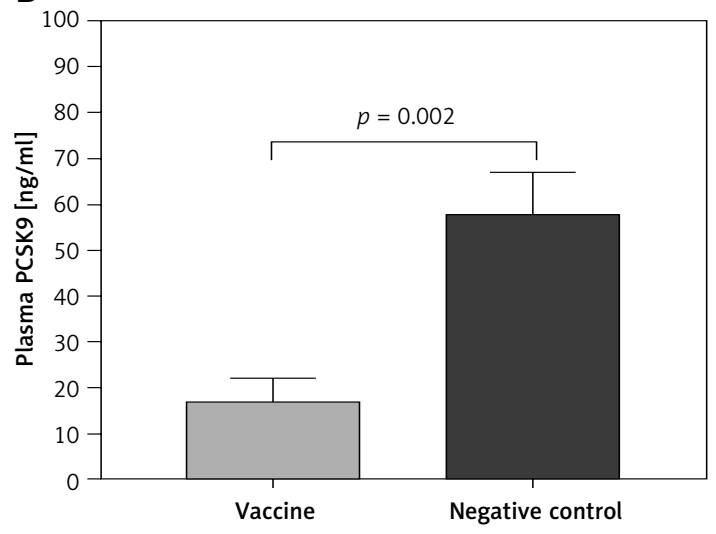

D

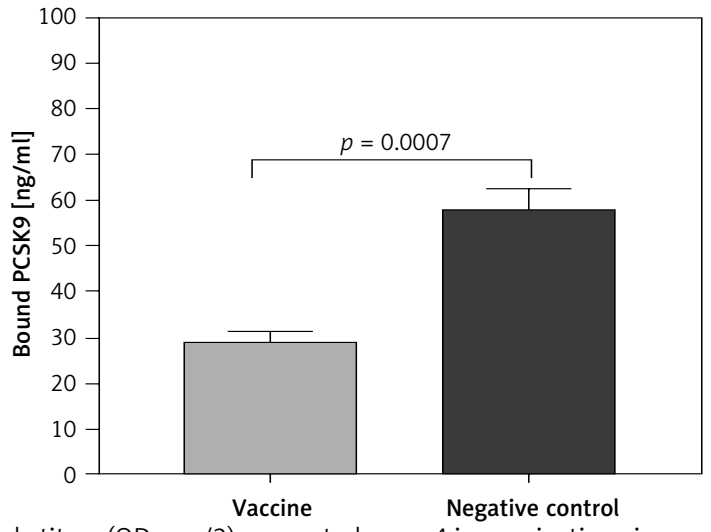

Figure 5. AntiPCSK9 vaccine efficacy. A - AntiPCSK9 antibody titres (ODmax/2) generated upon 4 immunisations in a bi-weekly interval (signed by arrows) have been evaluated upon 12 weeks post prime immunisation. B - Plasma levels of PCSK9 in the vaccine and control group were $17 \pm 5 \mathrm{ng} / \mathrm{ml}$ and $58 \pm 9 \mathrm{ng} / \mathrm{ml}$, respectively. C - Direct detection of antibodies bound to plasma PCSK9 in plasma samples from vaccinated and control mice. Increased $\mathrm{OD}_{450}$ is indicative for vaccine-generated anti-PCSK9 antibodies, which directly target PCSK9. D - In vitro PCSK9/LDLR binding assay. Plasma sample of vaccine group could decrease PCSK9 binding to LDLR by 50\%, when compared with plasma sample of control group. Values are expressed as means \pm SD $(n=3$ replicates of the pooled samples of 10 mice per group). Significance compared to control values was analysed by unpaired 2-tailed Student's $t$-test 
Efficacy study in mice bearing B16F0 melanoma tumours

The effect of PSCK9 inhibition on skin cancer progression was evaluated using a model of B16F0 melanoma tumour developed in the vaccinated C57BL/6 mice. The protective effect of the liposomal-antiPCSK9 vaccine on tumour-bearing mice was studied by monitoring body weight alterations, tumour growth rate in terms of mean tumour size $\left(\mathrm{mm}^{3}\right)$, and survival. The weight monitoring curve (Figure $6 \mathrm{~A}$ ) and the integrated areas under the body weight curve $\left(A \cup C_{\text {body weight }}\right)$ over 36 days (Figure 6 B) showed that body mass was not significantly different between the vaccine, liposomal doxorubicin (nanoliposomal doxorubicin), and control group. To determine the endpoint therapeutic potential of the liposomal-antiPCSK9 vaccine in tumour-bearing C57BL/6 mice, time to reach endpoint (TTE), and percentage of tumour growth delay (\%TGD), as well as median survival time (MST) and increase life span (ILS) were measured (Table III). As revealed by TTE and \%TGD, tumour growth was not significantly different between the vaccine, liposomal doxorubicin, and control groups (Figure 7 and Table III). TTE values of the vaccine, liposomal doxorubicin, and control groups were $28 \pm 5,34 \pm 2$, and $31 \pm 2$ days, respec- tively. Percentage TGD values of the vaccine, Doxil, and control groups were $11.5 \pm 15.4 \%, 7.75 \pm 6.5 \%$, and $0 \pm 7.5$, respectively.

Kaplan-Meier curves (Figure 8) showed that the survival of both the vaccine and liposomal doxorubicin treated groups was not significantly different from the control mice ( $p>0.05$, log-rank test). The MST of the vaccine, liposomal doxorubicin, and control group was 32, 35, and 32 days, respectively. Further analysis did not reveal any difference between the ILS indices of the vaccinated group and the other groups (Table III).

To evaluate the effect of PCSK9 inhibition on tumour chemotherapy, a vaccinated group were taken under single-dose Liposomal doxorubicin treatment (called the combination group) and compared with the group only vaccinated and with the group treated with liposomal doxorubicin alone. It was found that body weight change was not significantly different between the combination, vaccine, liposomal doxorubicin, and control groups (Figures $6 \mathrm{~A}$ and B). Values of TTE and TGD\% revealed that the tumour volume in the combination group was not significantly different from the vaccine, liposomal doxorubicin, and control group (Figure 7 and Table III). Kaplan-Meier curve analysis using log-rank test showed that
A

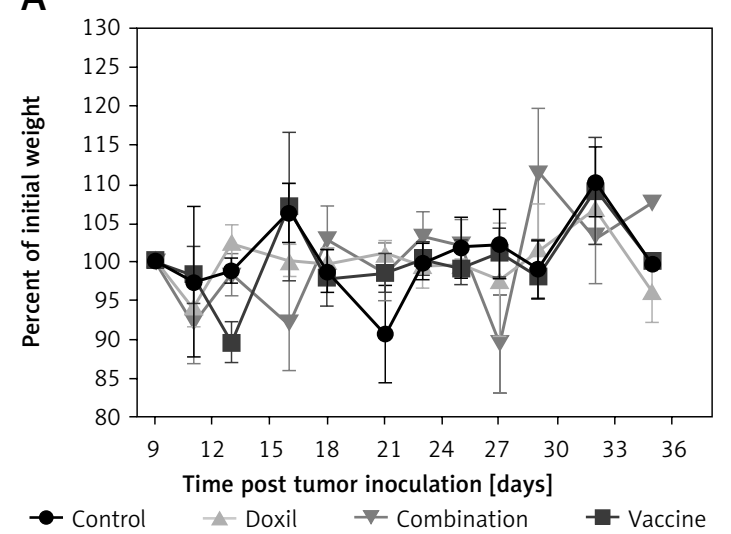

B

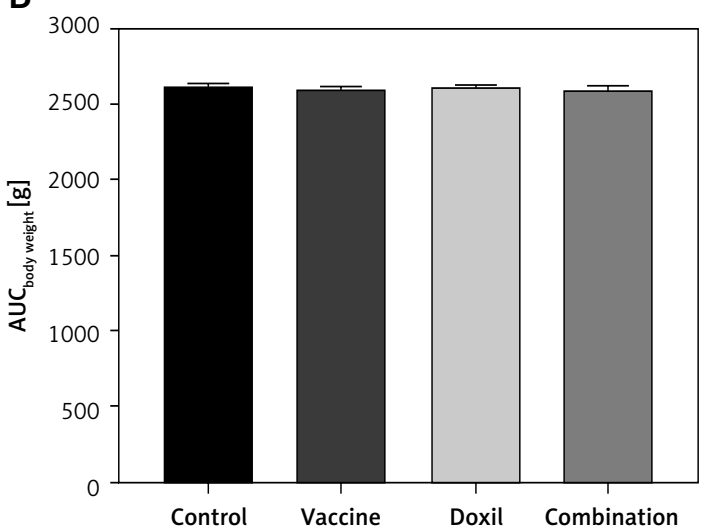

Figure 6. A - The weight monitoring curve exhibits point by point alterations of the body weight during 36 days in the control, vaccine, and Doxil group. B - The integrated areas under the body weight curve (AUC ${ }_{\text {ody weight }}$ ) over 36 days demonstrate overall weight changes. The body weight loss was not significantly different between the vaccine, Doxil, and the control group. Animal body weight was measured every 3 days. Data are presented as the mean $\pm \mathrm{SD}(n=8) . P<0.05$ was considered as the level of statistical significance

Table III. Therapeutic efficacy data of different treatments in C57BL/6 mice bearing B16F0 tumour

\begin{tabular}{|lcccc|}
\hline Groups & TTE $^{\mathrm{a}}$ (mean \pm SD) [days] & TGD $^{\mathrm{b}}(\%)$ & MST $^{\mathrm{c}}$ [days] & ILS $^{\mathrm{d}}$ (\%) \\
\hline Control & $31 \pm 2$ & - & 32 & - \\
\hline Vaccine & $28 \pm 5$ & -11.5 & 32 & 0 \\
\hline Doxil & $34 \pm 2$ & 7.8 & 35 & 9.4 \\
\hline Combination & $30 \pm 6$ & -6.1 & 32 & 0 \\
\hline
\end{tabular}

aTime to reach end point, ${ }^{b}$ tumour growth delay, ${ }^{c}$ median survival time, ${ }^{d}$ increase in lifespan. 
A

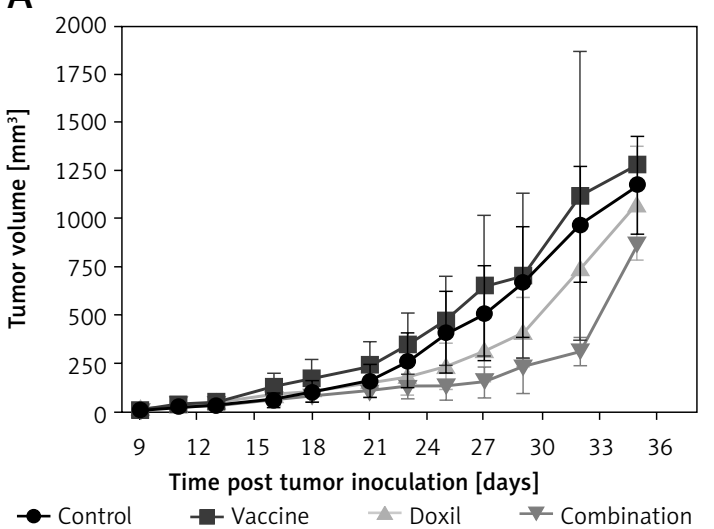

C

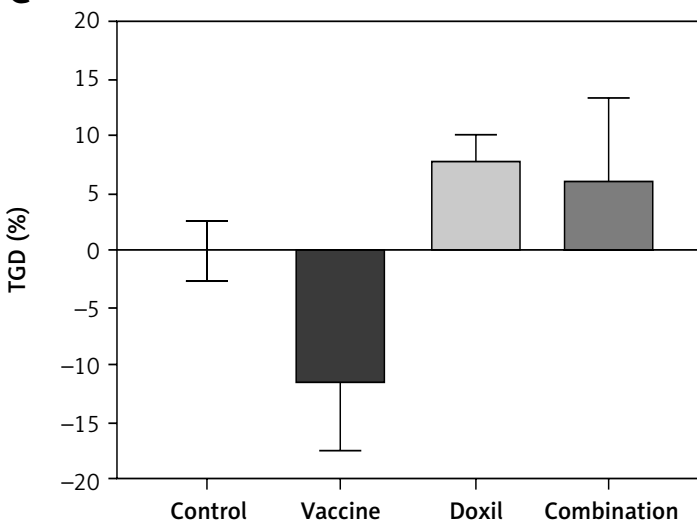

there was no significant difference in MST and ILS indices between the combination group and the other groups (Figure 8 and Table III).

\section{Discussion}

We recently reported that L-IFPTA ${ }^{+}$vaccine can effectively inhibit PCSK9 function through suppression of PCSK9/LDLR interaction via induction of antiPCSK9 antibodies in vaccinated BALB/C mice [41]. Although disrupted cholesterol homeostasis is known to play an important role in melanoma and other cancer types [1-10], the effects of lipid lowering agents, such as PCSK9 inhibitors, on cancer progression are unclear. Our previous experimental studies showed that L-IFPTA ${ }^{+}$vaccine-mediated PCSK9 inhibition not only caused no harmful effects but also could moderately suppress tumour growth and improve lifespan and survival in mice bearing breast [51] and colon cancers [52]. Importantly, it has been found that cholesterol can exert differing effects dependent on cancer type; therefore, evaluating the effect of PCSK9 inhibition on other cancer types [53], e.g. melanoma, is necessary.

The findings of the present study revealed that L-IFPTA $^{+}$vaccine could provoke functional anti-PCSK9 antibodies (Figure 5 A) that reduce plasma level and activity of PCSK9 (Figures 5 B and D)
B

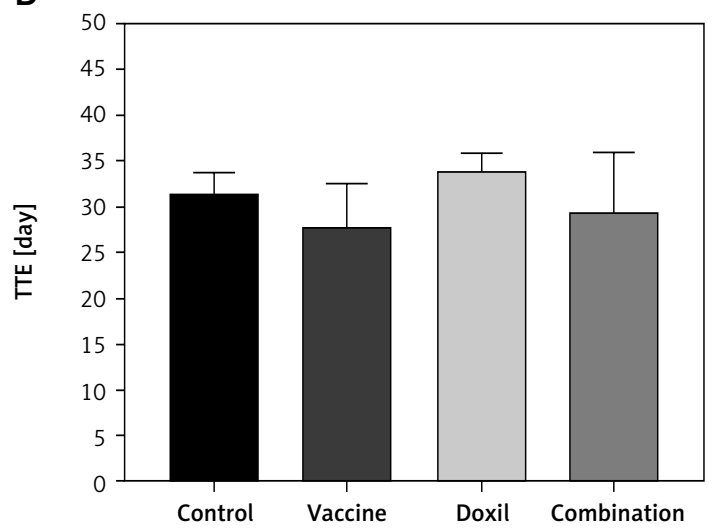

Figure 7. The tumour growth curve (A) shows increase of tumour size during 36 days in the control, vaccine, and Doxil group. Measuring the time to reach endpoint (TTE) (B) and tumour growth delay (TGD) (C) showed no significant difference between the studied groups. Tumour volume $\left(\mathrm{mm}^{3}\right)$ was measured every 3 days. Data are presented as the mean \pm SD $(n=8)$. Results with $p<0.05$ were considered as statistically significant

through specific and direct targeting of PCSK9 (Figure 5 C) in C57BL/6 mice. However, the L-IFPTA ${ }^{+}$ vaccine could not inhibit tumour growth (Figure 7 ), nor did it prolong survival (Figure 8 ) in melanoma tumour-bearing mice. When compared with the liposomal doxorubicin and control groups, PCSK9 vaccination did not change TTE, TGD\%, MST, or ILS, suggesting that L-IFPTA ${ }^{+}$vaccine did not exacerbate tumour behaviour and lifespan of mice bearing melanoma.

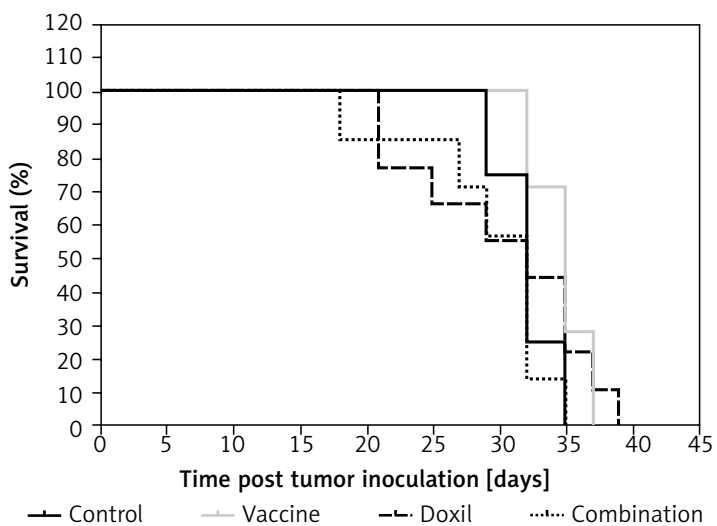

Figure 8. Kaplan-Meier curves exhibit the survival rate of the control, vaccine, and Doxil group. There was no significant difference between the compared groups. Data are presented as the mean \pm SD $(n=8) . P<0.05$ was considered as the level of statistical significance 
Although there is no evidence on the effects of PCSK9 inhibitors in cancerous conditions, recent studies have shown that PCSK9 variants with LDL-lowering mutations mimicking PCSK9 inhibitors are associated with a lower risk and occurrence of cancer $[36,54]$. Such inconsistency between results of our study and others is probably due to different modalities of PCSK9 inhibition. We inhibited circulating PCSK9, in which the LDLR-dependent effect of PCSK9 was affected, while genetic mutations possessing LDL-lowering variants can modulate intracellular activity of PCSK9 beyond its regulatory effect on LDLR, such as regulation of cell cycle and apoptosis [55, 56]. Additionally, L-IFPTA ${ }^{+}$vaccine, when combined with liposomal doxorubicin chemotherapy, did not aggravate the effects of chemotherapy nor improved the anti-tumour effect of liposomal doxorubicin. There was also no significant difference in lifespan and MST of mice in the vaccine and combination groups.

In conclusion, although L-IFPTA ${ }^{+}$vaccine-mediated PCSK9 inhibition could not decrease the growth of melanoma tumours and improve the survival of tumour-bearing animals, it did not aggravate tumour behaviour in melanoma-bearing mice either alone or when combined with chemotherapy (liposomal doxorubicin). Importantly, further preclinical and clinical investigations are needed to confirm the efficacy and safety of therapeutic inhibition of PCSK9 in patients with different types of cancer.

\section{Acknowledgments}

Amir Abbas Momtazi-Borojeni and Maryam Ebrahimi Nik equally contributed as the first author.

We are thankful for the financial support from the Mashhad University of Medical Sciences, Mashhad, Iran (grant number: 941726) and the National Institute for Medical Research Development (NIMAD), Tehran, Iran (grant nunber 963401)

\section{Conflict of interest}

Dr. Banach has served on a speaker's bureau and as an advisory board member for Amgen, Sanofi-Aventis, and Lilly. Other authors declare no conflict of interest.

\section{References}

1. Llaverias G, Danilo C, Mercier I, et al. Role of cholesterol in the development and progression of breast cancer. Am J Pathol 2011; 178: 402-12.

2. Li C, Yang L, Zhang D, Jiang W. Systematic review and meta-analysis suggest that dietary cholesterol intake increases risk of breast cancer. Nutr Res 2016; 36: 627-35.
3. Du O, Wang O, Fan $\mathrm{H}$, et al. Dietary cholesterol promotes AOM-induced colorectal cancer through activating the NLRP3 inflammasome. Biochem Pharmacol 2016; 105: 42-54.

4. Moon H, Ruelcke JE, Choi E, et al. Diet-induced hypercholesterolemia promotes androgen-independent prostate cancer metastasis via IQGAP1 and caveolin-1. Oncotarget $2015 ; 6: 7438$.

5. Radišauskas R, Kuzmickienè I, Milinavičienè E, Everatt R. Hypertension, serum lipids and cancer risk: a review of epidemiological evidence. Medicina 2016; 52: 89-98.

6. Pascual G, Avgustinova A, Mejetta S, et al. Targeting metastasis-initiating cells through the fatty acid receptor CD36. Nature 2017; 541: 41-5.

7. Ding $X$, Zhang W, Li S, Yang H. The role of cholesterol metabolism in cancer. Am J Cancer Res 2019; 9: 219-27.

8. Carvalho MA, Zecchin KG, Seguin F, et al. Fatty acid synthase inhibition with Orlistat promotes apoptosis and reduces cell growth and lymph node metastasis in a mouse melanoma model. Int J Cancer 2008; 123: 2557-65.

9. Xia S, Lin R, Jin L, et al. Prevention of dietary-fat-fueled ketogenesis attenuates BRAF V600E tumor growth. Cell Metabolism 2017; 25: 358-73.

10. Kwan HY, Fu X, Liu B, et al. Subcutaneous adipocytes promote melanoma cell growth by activating the Akt signaling pathway role of palmitic acid. J Biol Chem 2014; 289: 30525-37.

11. Huang P, Nedelcu D, Watanabe M, et al. Cellular cholesterol directly activates smoothened in hedgehog signaling. Cell 2016; 166: 1176-87e14.

12. Luchetti G, Sircar R, Kong JH, et al. Cholesterol activates the G-protein coupled receptor Smoothened to promote Hedgehog signaling. Elife 2016; 5: e20304.

13. Guixà-González R, Albasanz JL, Rodriguez-Espigares I, et al. Membrane cholesterol access into a G-protein-coupled receptor. Nat Commun 2017; 8: 14505.

14. Hooper JE, Scott MP. Communicating with hedgehogs. Nat Rev Mol Cell Biol 2005; 6: 306-17.

15. Sheng R, Chen Y, Gee HY, et al. Cholesterol modulates cell signaling and protein networking by specifically interacting with PDZ domain-containing scaffold proteins. Nat Commun 2012; 3: 1249.

16. Vaquero J, Ho-Bouldoires TN, Claperon A, Fouassier L. Role of the PDZ-scaffold protein NHERF1/EBP50 in cancer biology: from signaling regulation to clinical relevance. Oncogene 2017; 36: 3067-79.

17. Lavigne PM, Jafri $H$, Karas R. The association between lower levels of low-density lipoprotein cholesterol and cancer predates the diagnosis of cancer by 18 years. J Am Coll Cardiol 2012; 59 (13 Suppl): E1622.

18. Jacobs EJ, Newton CC, Thun MJ, Gapstur SM. Long-term use of cholesterol-lowering drugs and cancer incidence in a large United States cohort. Cancer Res 2011; 71: 1763-71.

19. Murtola TJ, Visvanathan K, Artama M, Vainio H, Pukkala E. Statin use and breast cancer survival: a nationwide cohort study from Finland. PLoS One 2014; 9: e110231.

20. Cardwell CR, Hicks BM, Hughes C, Murray LJ. Statin use after colorectal cancer diagnosis and survival: a population-based cohort study. J Clin Oncol 2014; 32: 3177-83.

21. Nielsen SF, Nordestgaard BG, Bojesen SE. Statin use and reduced cancer-related mortality. N Engl J Med 2012; 367: 1792-802.

22. Abifadel $M$, Varret $M$, Rabès JP, et al. Mutations in PCSK9 cause autosomal dominant hypercholesterolemia. Nat Genet 2003; 34: 154-6. 
23. Cohen J, Pertsemlidis A, Kotowski IK, Graham R, Garcia $\mathrm{CK}$, Hobbs HH. Low LDL cholesterol in individuals of Af rican descent resulting from frequent nonsense mutations in PCSK9. Nat Genet 2005; 37: 161-5.

24. Cohen JC, Boerwinkle E, Mosley Jr TH, Hobbs HH. Se quence variations in PCSK9, low LDL, and protection against coronary heart disease. N Engl J Med 2006; 354 1264-72.

25. Zhao Z, Tuakli-Wosornu Y, Lagace TA, et al. Molecular characterization of loss-of-function mutations in PCSK9 and identification of a compound heterozygote. Am J Hum Genet 2006; 79: 514-23.

26. Hooper AJ, Marais AD, Tanyanyiwa DM, Burnett JR. The C679X mutation in PCSK9 is present and lowers blood cholesterol in a Southern African population. Atherosclerosis 2007; 193: 445-8.

27. Food U. Administration D. FDA approves Repatha to treat certain patients with high cholesterol. 2015

28. Sabatine MS, Giugliano RP, Wiviott SD, et al. Efficacy and safety of evolocumab in reducing lipids and cardiovas cular events. N Engl J Med 2015; 372: 1500-9.

29. Sahebkar A, Watts GF. New therapies targeting apoB metabolism for high-risk patients with inherited dyslipidaemias: what can the clinician expect? Cardiovasc Drugs Ther 2013; 27: 559-67.

30. Dragan S, Serban MC, Banach M. Proprotein convertase subtilisin/kexin 9 inhibitors: an emerging lipid-lowering therapy? J Cardiovasc Pharmacol Ther 2015; 20: 157-68.

31. Jaworski K, Jankowski P, Kosior DA. PCSK9 inhibitors from discovery of a single mutation to a groundbreaking therapy of lipid disorders in one decade. Arch Med Sci 2017; 13: 914-29.

32. Macchi C, Banach M, Corsini A, Sirtori CR, Ferri N, Rus cica $M$. Changes in circulating pro-protein convertase subtilisin/kexin type 9 levels - experimental and clinical approaches with lipid-lowering agents. Eur J Prev Cardiol 2019; 2047487319831500.

33. Soran H, Adam S, Mohammad JB, et al. Hypercholesterolaemia - practical information for non-specialists. Arch Med Sci 2018; 14: 1-21.

34. Benn M, Tybjærg-Hansen A, Stender S, Frikke-Schmidt R, Nordestgaard BG. Low-density lipoprotein cholesterol and the risk of cancer: a mendelian randomization study. J Natl Cancer Instit 2011; 103: 508-19.

35. Folsom AR, Peacock JM, Boerwinkle E. Sequence variation in proprotein convertase subtilisin/kexin type 9 serine protease gene, low LDL cholesterol, and cancer incidence. Cancer Epidemiol Prev Biomarkers 2007; 16 2455-8.

36. Nowak C, Ärnlöv JA. Mendelian randomization study of the effects of blood lipids on breast cancer risk. Nat Commun 2018; 9: 3957

37. Galabova G, Brunner S, Winsauer G, et al. Peptide-based anti-PCSK9 vaccines-an approach for long-term LDLC management. PLoS One 2014; 9: e114469.

38. Fattori E, Cappelletti M, Surdo PL, et al. Immunization against proprotein convertase subtilisin-like/kexin type 9 lowers plasma LDL-cholesterol levels in mice. J Lipid Res 2012; 53: 1654-61.

39. Crossey E, Amar MJ, Sampson M, et al. A cholesterol-lowering VLP vaccine that targets PCSK9. Vaccine 2015; 33: 5747-55.

40. Landlinger C, Pouwer MG, Juno C, et al. The AT04A vac cine against proprotein convertase subtilisin/kexin type 9 reduces total cholesterol, vascular inflammation, and atherosclerosis in APOE* 3 Leiden. CETP mice. Eur Heart 2017; 38: 2499-507.
41. Momtazi-Borojeni AA, Jaafari MR, Badiee A, Sahebkar A. Long-term generation of antiPCSK9 antibody using a nanoliposome-based vaccine delivery system. Atherosclerosis 2019; 283: 69-78.

42. Momtazi-Borojeni AA, Jaafari MR, Badiee A, Sahebkar A. Long-term generation of antiPCSK9 antibody using a nanoliposome-based vaccine delivery system. Atherosclerosis 2019; 283: 69-78.

43. Galabova G, Brunner S, Winsauer G, et al. Peptide-based anti-PCSK9 vaccines-an approach for long-term LDLC management. PLoS One 2014; 9: e114469.

44. Schneeberger A, Mandler M, Otava O, et al. Development of AFFITOPE vaccines for Alzheimer's disease (AD) - from concept to clinical testing. J Nutr Health Aging 2009; 13: 264-7.

45. Slingluff CL, Yamshchikov G, Neese P, et al. Phase I trial of a melanoma vaccine with gp100280-288 peptide and tetanus helper peptide in adjuvant: immunologic and clinical outcomes. Clin Cancer Res 2001; 7: 3012-24.

46. Bartlett GR. Phosphorus assay in column chromatography. J Biol Chem 1959; 234: 466-8.

47. Huang Z, Szoka Jr FC. Sterol-modified phospholipids: cholesterol and phospholipid chimeras with improved biomembrane properties. J Am Chem Soc 2008; 130: 15702-12.

48. Huang Z, Jaafari MR, Szoka Jr FC. Disterolphospholipids: nonexchangeable lipids and their application to liposomal drug delivery. Angewandte Chemie 2009; 121: 4210-3.

49. Schluep T, Hwang J, Cheng J, et al. Preclinical efficacy of the camptothecin-polymer conjugate IT-101 in multiple cancer models. Clin Cancer Res 2006; 12: 1606-14.

50. Huang SK, Mayhew E, Gilani S, Lasic DD, Martin FJ, Papahadjopoulo, D. Pharmacokinetics and therapeutics of sterically stabilized liposomes in mice bearing C-26 coIon carcinoma. Cancer Res 1992; 52: 6774-81.

51. Momtazi-Borojeni AA, Nik ME, Jaafari MR, Banach $M$, Sahebkar A., Effects of immunization against PCSK9 in an experimental model of breast cancer. Arch Med Sci 2019; 15: 570-9.

52. Momtazi-Borojeni AA, Nik ME, Jaafari MR, Banach $M$, Sahebkar A. Potential anti-tumor effect of a nanoliposomal antiPCSK9 vaccine in mice bearing colorectal cancer. Arch Med Sci 2019; 15: 559-69.

53. Swinnen JV, Brusselmans K, Verhoeven G. Increased lipogenesis in cancer cells: new players, novel targets. Curr Opin Clin Nutr Metabol Care 2006; 9: 358-65.

54. Schmidt AF, Holmes MV, Preiss D, et al. Phenome-wide association analysis of LDL-cholesterol lowering genetic variants in PCSK9. bioRxiv 2018; 329052.

55. Lan H, Pang L, Smith MM, et al. Proprotein convertase subtilisin/kexin type 9 (PCSK9) affects gene expression pathways beyond cholesterol metabolism in liver cells. J Cell Physiol 2010; 224: 273-81.

56. Ranheim T, Mattingsdal M, Lindvall JM, et al. Genome-wide expression analysis of cells expressing gain of function mutant D374Y-PCSK9. J Cell Physiol 2008; 217: 459-67. 